\title{
Effect of Acupuncture Transcutaneous Electrical Nerve Stimulation on Upper Limb Function in Stroke Patients
}

\author{
MARAM A. MAHMOUD, M.Sc.*; EMAN S. FAYEZ, Ph.D.**; MAGED M. ABDEL NASEER, M.D.*** and \\ RANIA M. TAWFIK, Ph.D.** \\ The Department of Physical Therapy, Emergency Hospital, Cairo University Hospitals*, \\ The Department of Physical Therapy for Neuromuscular Disorders and its Surgery, Faculty of Physical Therapy** and \\ Neurology, Faculty of Medicine***, Cairo University
}

\begin{abstract}
Background: Stroke is the leading cause of long-term disability; the restoration of movements and associated functions is a common concern of stroke patients. However, the restoration of the upper limb function is often poor, being seen in less than half of patients. Electrical stimulation is shown to be effective in enhancing the upper extremity functional recovery in stroke patients.
\end{abstract}

Aim of Study: To determine the effect of acupuncture TENS on upper limb function in stroke patients.

Procedures: This study was conducted on thirty male stroke patients. Patients were randomly assigned into two equal groups: The study group (GI) and the control group (GII). Group I (study group): This group received acupuncture (TENS) in addition to the selected physical therapy program. Group II (control group): This group received the selected physical therapy program only. Fugel-Meyer Scale was used to assess the upper limb function and Modified Ashworth scale (MAS) was used to assess the spasticity grades before and after four weeks of the treatment program.

Results: The results showed that: There was a significant difference of Fugel-Meyer Scale scores and mean values of spasticity grades in study group after the designed treatment program.

Conclusion: The acupuncture Transcutaneous Electrical Nerve Stimulation (TENS) has a significant effect in reduction of spasticity and improvement of upper extremity function following stroke.

Key Words: Acupuncture - Transcutaneous electric nerve stimulation - Electro acupuncture - Stroke Upper limb function.

\section{Introduction}

A STROKE, or Cerebrovascular Accident (CVA), is the rapid loss of brain function due to disturbance in the blood supply to the brain.

Correspondence to: Dr. Maram A. Mahmoud, The Department of Physical Therapy, Emergency Hospital, Cairo University Hospitals
Stroke is defined by the World Health Organization (WHO) as a "rapidly developing syndrome with clinical signs of focal or global disturbance of cerebral function, with symptoms lasting 24 hours or longer or leading to death, with no apparent cause other than vascular origin" [1].

Stroke is the leading cause of long-term disability worldwide; the restoration of movements and associated functions is a common concern of stroke patients. However, the restoration of the upper limb movements and functions is often poor, generally being seen in less than half of patients [2].

Spasticity is a component of upper-motor neuron syndromes such as stroke, and has been defined as a motor disorder characterized by a velocitydependent increase in tonic stretch reflexes with exaggerated tendon jerks, which result from the hyper excitability of the stretch reflex. Severe spasticity can cause reduced flexibility, posture, and functional mobility, as well as joint pain, contracture, and difficulty in positioning for comfort and hygiene [3] .

The upper extremity function is impaired after stroke in approximately $70-80 \%$ of patients in acute phase and in $40 \%$ in chronic phase. This impairment limits the voluntary, well-coordinated, and effective movements as well as a person's level of activity and participation in their social and physical environment. This longstanding disability might also influence the quality of life [4]

Paresis of the upper limb is the most common impairment after stroke. It is caused by a reduction of the ability to voluntarily activate the motor neurons [5]. 
Acupuncture is one of the main modes of treatment in Traditional Chinese Medicine (TCM), and has been performed for more than 2,000 years. It was widely used to treat hemiplegia long before the Tang dynasty. TCM is based on the yin-yang theory, where energy called chi flows through the organs is essential in maintaining good health. Acupuncture can correct the imbalance of energy circulation and could improve post stroke motor ability and might be a useful supplement to stroke rehabilitation [3].

Acupuncture has been used for thousands of years as an effective therapy for regulating pain and managing many other functions of the body. A number of different types of acupuncture have come into use over the centuries. Electro Acupuncture (EA) has been shown to significantly enhance traditional acupuncture treatments [6]

TENS applied repetitively to the acupuncture points for 3-4 weeks has been found to reduce clinical spasticity and improve motor functions in chronic hemiplegic stroke. Possible mechanisms could be partly attributable to an enhancement of presynaptic inhibition of the hyperactive stretch reflexes in spastic muscles, disinhibition of descending voluntary commands to the motor neurons of the paretic muscles, and decrease in cocontraction of the spastic antagonist [7].

This study was designed to determine the effect of acupuncture TENS on upper limb function in stroke patients.

\section{Patients and Methods}

\section{Patients' selection:}

A Randomized Controlled Trial (RCT) included thirty male stroke patients. Patients were selected from the outpatient clinic of the Faculty of Physical Therapy, Cairo University and Kasr El-Ainy Hospital in the period from May 2018 to November 2018. The patients were diagnosed as having stroke in the domain of carotid system based on careful clinical assessment by a neurologist and radiological investigations including computed axial tomography and/or magnetic resonance imaging of the brain. Patients were randomly assigned into two equal groups: The study group (GI) and the control group (GII).

- Group I (study group): This group included 15 males. Patients in this group received electro acupuncture (TENS) in addition to the selected physical therapy program.
- Group II (control group): This group included 15 males. Patients in this group received the selected physical therapy program only.

Inclusion criteria were: Duration of illness before participation in the study ranged from six to eighteen months post stroke, patients' age ranged from 45-60 years, patients with sufficient cognitive abilities that enables them to understand and follow instructions (mini-mental scale >24) (Appendix I), patients with mild spasticity of the affected upper limb ranged from 1 to $1+$ according to Modified Ashworth scale.

Exclusion criteria include: Patients with communication problems, patients with a history of previous stroke or other neurological diseases or disorders, patients with inflammation, old fracture or prosthesis in the affected limb, neurological diseases that affect upper limb function other than stroke (eg: Multiple sclerosis, Peripheral neuropathy, Parkinsonism .... etc.), infection or dermatological conditions on acupuncture sites, musculoskeletal disorders such as severe arthritis, cardiovascular problems (unstable angina, recent myocardial infarction within the last three months, congestive heart failure, significant heart valve dysfunction, arrhythmia, implanted pacemaker or unstable hypertension) or pulmonary disorders, visual and auditory problems and patients with cognitive impairment.

\section{Instrumentations:}

\section{Instrumentations used for assessment:}

1-Modified Ashworth scale: The Modified Ashworth scale (MAS) is a 6-point rating scale that was used to measure muscle tone. The scale is the most widely used method in clinical practice and research field. The Ashworth Scale has good intra-and interrater reliability in patients with upperlimb spasticity after stroke [8]

2- FUGL-MEYER upper extremity (FMUE) scale: The Fugl-Meyer Upper Extremity Scale is a widely used and highly recommended strokespecific, performance-based measure of impairment. It is designed to assess reflex activity, movement control and muscle strength in the Upper Extremity (UE) of people with post stroke hemiplegia. The UE subscale of the Fugl-Meyer Assessment (FMA) is a validated reliable index measuring motor impairment in stroke patients. The FMUE Scale comprises 33 items, each scored on a scale of 0 to 2 , where $0=$ cannot perform, $1=$ performs partially and $2=$ performs fully. It is free, requires only household items for testing, and takes up to 30 minutes to administer [9]. 


\section{Instrumentations used for treatment:}

\section{Electrical stimulation device (TENS):}

Device model (TENS 210, Made in USA and carry a serial no. 45TE0033) was used in the current study, this apparatus was used to deliver TENS current with a frequency of $100 \mathrm{~Hz}$. The intensity of the current was increased to the point where the patient reported. At this point, the current was slightly reduced to an unpleasant but tolerable intensity. Electrical stimulation time was $20 \mathrm{~min}$ utes.

\section{Procedures:}

\section{A-Assessment protocol:}

1- Prior to participation in this study, the aim and procedures were explained to the patients and a written consent was obtained from all patients (Appendix IV).

2- History taking and neurological examination according to the neurological sheet of department of neurology, Kasr Al-Ainy Hospitals were done for all patients.

3-Assessment of muscle tone: Muscle tone was tested for shoulder adductors, elbow flexors, wrist flexors, and finger flexors. The patient assumes a comfortable supine lying position, with his head in mid position. The therapist passively moved the shoulder joint through the available ROM (in abduction and adduction) then elbow joint, wrist joint and the MCP joints through the available ROM (in flexion and extension) trying to estimate the amount of resistance that is felt according to Modified Ashworth Scale. This test was performed pre and post-treatment for both groups [8]. Appendix (II).

4- Assessment of upper limb function by FuglMeyer upper extremity scale: The patients were examined for upper limb function using FuglMeyer upper extremity scale pre and posttreatment. The five domains assessed by FuglMeyer scale are: Motor function (maximum score in upper limb $=66$, maximum score in lower limb=34), sensory function (maximum score $=24)$, balance $($ maximum score $=14)$, range of motion of joints (maximum score $=44$ ) and Joint pain (maximum score $=44$ ). The maximum total score that could be obtained in Fugl Meyer assessment is 226 , though it is common practice to assess all domains separately. The test could be completed in around 40 minutes. To perform the test, we needed a tennis ball, a small spherical shaped container and knee hammer [9]. Appendix (III).

\section{B- Treatment protocol:}

Group I: Patients in this group received electro acupuncture (TENS) in addition to a selected physical therapy program.

\section{Application of TENS:}

\section{Identification of acupuncture points:}

The electrodes were applied on the following acupuncture points on the subject's hemiparetic arm respectively (Zeqian (EX-UE, A32, Shounizhu (EX-UE),) Shaohai (HT3) and Neiguan (PC6)). Fig. (1).

These points were selected for the treatment and were identified according to (Wang et al. [3] protocol. Table (1).

Table (1): Acupuncture points location depth [3]

\begin{tabular}{|c|c|}
\hline $\begin{array}{l}\text { 1- Neiguan } \\
\text { (PC6) }\end{array}$ & $\begin{array}{l}\text { - On the palmar side of the forearm, } 2 \text { cun above } \\
\text { the transverse crease of the wrist, between the } \\
\text { tendons of the palmaris longus muscle and flexor } \\
\text { carpi radialis muscle. } \\
\text { - Perpendicular insertion } 0.5-0.8 \text { cun. }\end{array}$ \\
\hline $\begin{array}{l}\text { 2- Shaohai } \\
\text { (hT3). }\end{array}$ & $\begin{array}{l}\text { - With the elbow flexed, at a midpoint of the line } \\
\text { connecting the medial end of cubital crease and } \\
\text { the medial epicondyle of the humerus. } \\
\text { - Perpendicular insertion } 0.5-0.8 \text { cun. }\end{array}$ \\
\hline $\begin{array}{l}\text { 3- Shounizhub } \\
\text { (ex-Ue) }\end{array}$ & $\begin{array}{l}\text { - Locate at the flexion side of the forearm, } 6 \text { cun } \\
\text { above the transverse crease of the wrist, between } \\
\text { the tendons of palmaris longus and flexor carpi } \\
\text { radialis muscle. } \\
\text { - Perpendicular insertion } 0.5-1.0 \text { cun. }\end{array}$ \\
\hline $\begin{array}{l}\text { 4- Zeqianb } \\
\text { (ex-Ue, A32) }\end{array}$ & $\begin{array}{l}\text { - } 1 \text { cun below Chize (IU5), which is at radial edge } \\
\text { of biceps tendon in elbow crease. } \\
\text { - Perpendicular insertion 0.5-1 cun. }\end{array}$ \\
\hline
\end{tabular}

Notes: Cun is a measurement used for acupuncture point location. One cun is equal to the space between the distal interphalangeal joint and the proximal interphalangeal joint on the middle finger.

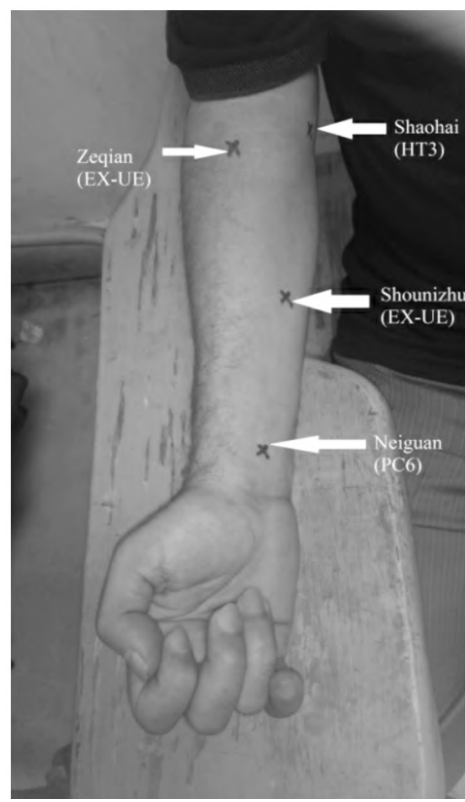

Fig. (1): Acupuncture points location depth. 


\section{B- Skin preparation:}

- The skin was inspected for any pre-existing irritation, cuts, scares or lesion.

- To increase conductance, the hair was moved away from the site of stimulation and the surface of the skin was cleaned to remove any signs of lotion, dirt, etc. and dried well.

\section{C- Stimulation procedures:}

Each patient seated in a comfortable chair with back support, the patient sit up with his back straight and his shoulders back, all 3 normal back curves should be present while sitting, distribute his body weight evenly on both hips, bended his knees at a right angle, keep his feet flat on the floor, shoulder relaxed elbow in 90 degree flextion, supinated forearm, wrist in neutral position (supported on arm seat).

- TENS was applied via surface electrodes $(5 \mathrm{~cm}$ $X 7 \mathrm{~cm}$ ), positioned on acupuncture points.

- The stimulation frequency was $100 \mathrm{~Hz}$.

- The intensity of the current was increased to the point where the patient reported. At this point, the current was slightly reduced to an unpleasant but tolerable intensity.

- The duration of session was 20 minutes. It was performed 3 times/week for 4 weeks. Fig. (2).

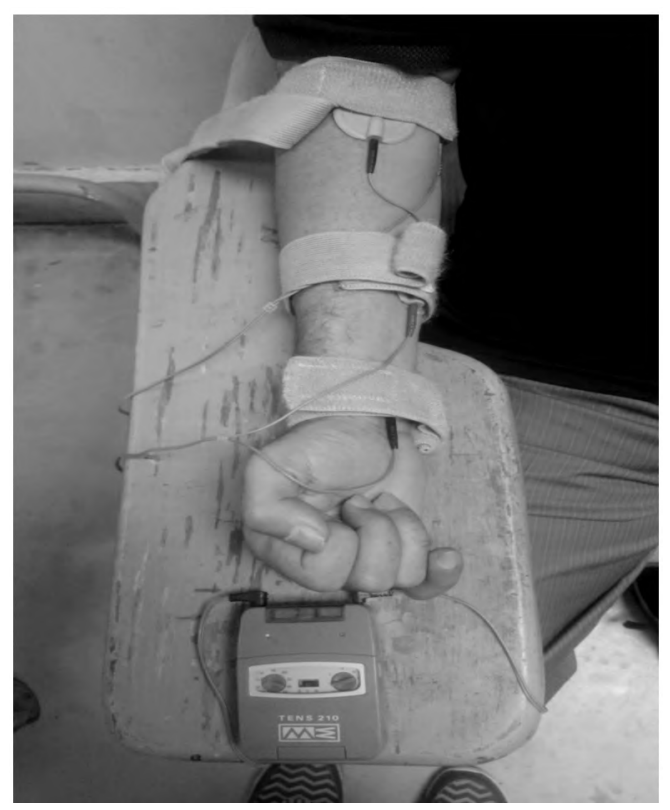

Fig. (2): Application of acupuncture TENS.

Group II: Patients in this group received the selected physical therapy program only.

The selected physical therapy program includes the following: Facilitation for weak muscles:
Through local facilitatory techniques (muscle tapping, vibration), strengthening exercise: For weak muscles which have grade "three" or above in power, range-of-motion exercises for the hemiparetic side, postural control and balance activities, upper extremity control, Proprioceptive Neuromuscular Facilitation (PNF) and Weight bearing and weight shift exercise.

\section{Statistical analysis:}

Data was compiled and analyzed using statistical SPSS software for windows, descriptive analysis: Mean and standard deviation for age, weight, height, paired $t$-test: For pre and post-treatment comparisons within control and study group, unpaired $t$-test: For comparison of fugel scale scores between pre-and post-treatment values of both control and study groups. $p$-value was set to 0.05 , $p<0.05$ means significant difference, $p>0.05$ means non-significant difference.

\section{Results}

Demographic and clinical characteristics of the patients in both groups:

By comparing the general characteristics of the patients in both groups, the results showed that there was no significant difference between both groups regarding mean age, weight and height ( $p=$ $0.7703, p=0.3000$ and $p=0.2588$ respectively) (Table 2).

Table (2): General characteristics of the patients in both groups.

\begin{tabular}{lcccc}
\hline & $\begin{array}{c}\text { Group I } \\
\mathrm{X} \pm \mathrm{SD}\end{array}$ & $\begin{array}{c}\text { Group II } \\
\mathrm{X} \pm \mathrm{SD}\end{array}$ & $\begin{array}{c}p- \\
\text { value }\end{array}$ & Sig. \\
\hline Age (years) & $50.75 \pm 2.864$ & $51.88 \pm 2.525$ & 0.7703 & $\mathrm{NS}$ \\
Weight $(\mathrm{kg})$ & $67.33 \pm 2.730$ & $80.13 \pm 1.437$ & 0.3000 & $\mathrm{NS}$ \\
Height $(\mathrm{cm})$ & $168.2 \pm 1.962$ & $170.9 \pm 1.226$ & 0.2588 & $\mathrm{NS}$ \\
\hline
\end{tabular}

Mean values of Fugel Scale scores pre and post-treatment for control group:

The mean value of Fugel Scale scores for control group pre-treatment was $33.79 \pm 2.036$ while post-treatment was $37.14 \pm 2.038$, which means that there was no significant difference of Fugel Scale scores post-treatment ( $p=0.2544)$ as shown in (Table 3).

Table (3): Mean values of Fugel Scale scores pre and posttreatment for control group.

\begin{tabular}{|c|c|c|}
\hline Variables & $\begin{array}{c}\text { Fugel Scale scores (Control group) } \\
\text { Mean } \pm \text { S.D. }\end{array}$ & $p$-value \\
\hline Pre & $33.79 \pm 2.036$ & $1.1650 .2544(\mathrm{NS})$ \\
\hline Post & $37.14 \pm 2.038$ & \\
\hline
\end{tabular}

NS: Non-Significant. 
Mean values of Fugel Scale scores pre and post-treatment for study group:

The mean value of Fugel Scale scores for study group pre-treatment was $36.19 \pm 1.904$ while posttreatment was $46.31 \pm 1.870$, which means that there was a significant difference of Fugel Scale scores post-treatment $(p=0.0007)$ as shown in (Table 4).

Table (4): Mean values of Fugel Scale scores pre and posttreatment for study group.

\begin{tabular}{lccc}
\hline Variables & $\begin{array}{c}\text { Fugel Scale scores (Study group) } \\
\text { Mean } \pm \text { S.D. }\end{array}$ & $t$ & $p$-value \\
\hline Pre & $36.19 \pm 1.904$ & 3.793 & $0.0007^{*}$ \\
Post & $46.31 \pm 1.870$ & & \\
\hline
\end{tabular}

*: Significant $<0.05$

Comparison between pre-treatment mean values of Fugel Scale (control vs study group):

The mean value of Fugel Scale scores for control group pre-treatment was $35.88 \pm 2.298$, and for study group was $36.19 \pm 1.904$, which means that there was no significant difference of Fugel Scale scores in both groups pre-treatment $(p=0.9173)$ as shown in (Table 5).

Table (5): Mean values of Fugel Scale scores, pre-treatment for control and study group.

\begin{tabular}{lccc}
\hline Variable & $\begin{array}{c}\text { Fugel Scale scores (pre) } \\
\text { Mean } \pm \text { S.D. }\end{array}$ & $t$ & $p$-value \\
\hline Control & $35.88 \pm 2.298$ & 0.1047 & 0.9173 (NS) \\
Study & $36.19 \pm 1.904$ & & \\
\hline
\end{tabular}

NS: Non-Significant.

Comparison between post-treatment mean values of Fugel Scale scores (control vs study group):

The mean value of Fugel Scale scores for control group post-treatment was $53.31 \pm 1.901$ and for Study group was $62.94 \pm 1.657$, which means that there was a significant difference of Fugel Scale scores in study group post-treatment $(p=0.0006)$ as shown in (Table 6).

Table (6): Mean values of Fugel Scale scores, post-treatment for control and study group.

\begin{tabular}{lccc}
\hline Variable & $\begin{array}{c}\text { Fugel Scale scores (post) } \\
\text { Mean } \pm \text { S.D. }\end{array}$ & $t$ & $p$-value \\
\hline Control & $\begin{array}{l}37.56 \pm 1.958 \\
\text { Study }\end{array}$ & 3.232 & $0.0030^{*}$ \\
\hline
\end{tabular}

*: Significant $<0.05$.

Comparison between mean values of pre \& post-treatment scores for modified Ashworth scale, control vs study groups:

The mean value of modified Ashworth scale for control group patients was in pre-treatment value was $2.867 \pm 0.1652$ and for study group was $2.667 \pm 0.1869$ at $(p=0.4294)$, which means there was no significant difference of modified Ashworth Scale between both groups before applying the designed treatment programs (Table 7).

The mean value of modified Ashworth scale for control group patients was in pre-treatment value was $2.867 \pm 0.1652$ for post-treatment values was $2.400 \pm 0.1633$, at $(p=0.0543)$, which means there was no significant difference of Modified Ashworth Scale in control group after the designed treatment program (Table 7).

The mean value of modified Ashworth scale for study group patients was in pre-treatment value was $2.667 \pm 0.1869$ for post-treatment was $1.733 \pm$ 0.1182 , at $(p=0.0002 *)$, which means there was a significant difference of modified Ashworth scale in study group after the designed treatment program (Table 7).

The mean value of Modified Ashworth scale for control group patients was in post-treatment value was $2.400 \pm 0.1633$ for study group was 1.733 \pm 0.1182 at $\left(p=0.0026^{*}\right)$, which means there was a significant difference of modified Ashworth scale between both groups (improvement of study group) after applying the designed treatment programs (Table 7).

Table (7): Mean values of Modified Ashworth scale, pre and post values comparisons for control and study group.

\begin{tabular}{|c|c|c|c|c|}
\hline \multirow{2}{*}{ Variable } & \multicolumn{4}{|c|}{ Modified Ashworth Scale } \\
\hline & Pre & Post & $t$-test & $p$-value \\
\hline Control group & $2.867 \pm 0.1652$ & $2.400 \pm 0.1633$ & 2.009 & 0.0543 \\
\hline Study group & $2.667 \pm 0.1869$ & $1.733 \pm 0.1182$ & 4.221 & $0.0002 *$ \\
\hline$t$-test & 0.8018 & 3.307 & & \\
\hline$p$-value & 0.4294 & $0.0026^{*}$ & & \\
\hline
\end{tabular}

*: Significant $<0.05 . \quad$ NS: Non- Significant.

\section{Discussion}

In the present study, there was a statistically significant reduction in the post-treatment mean value of spasticity grades post-treatment in the study group while there was no significant difference in the median value of spasticity grades pre and post-treatment in control group.

The results of the current study come in agreement with Lim et al., [10] who indicated that acupuncture or electroacupuncture therapy is effective in reducing the spasticity after stroke. A subgroup analysis showed that acupuncture significantly decreased wrist, knee, and elbow spasticity in poststroke patients. They concluded that acupunc- 
ture or electroacupuncture could be effective in decreasing spasticity after stroke, but long-term studies are needed to determine the longevity of treatment effects.

Deshmukh et al., [11] performed a study on thirty subjects with sub-acute stroke of either side. TENS on acupuncture points was given in subjects of experimental group along with TRT (Task Related Training) and conventional program to evaluate the effectiveness of TENS. Measurement of spasticity was done by Modified Ashworth Scale (MAS), functional ability was measured by Dynamic Gait Index (DGI) and Timed Up \& Go (TUG) test. All the measurements were done before and after 5 weeks intervention. They observed a significant reduction in spasticity measured by MAS and relevant improvement in functional ability measured by DGI and TUG in experimental group after five weeks intervention.

Also, the results of the current study is supported by Zhao et al., [12] who performed a study on sixty patients with muscle spasticity after brain injury. The acupoints Hegu (LI4)-Yuji (LU10) and Zusanli (ST36)-Chengshan (BL57) on the injured side were stimulated at 0,2 , or $100 \mathrm{~Hz}, 5$ times per week for 4 weeks. The patients were followed-up for 1 and 2 months after the treatments. They observed that the wrist spasticity was significantly reduced from baseline at weeks 2,3 , and 4 of treatment and at the 1-and 2-month follow-up visits in the $100 \mathrm{~Hz}$ group. Compared with $2 \mathrm{~Hz}$ or sham TEAS, $100 \mathrm{~Hz}$ TEAS decreased wrist spasticity at weeks 2,3 , and 4 of treatment and 1 month after treatment. The other endpoints were not affected by the treatments. No treatment-emergent adverse events were reported during treatments and follow-up visits. They concluded that TEAS appears to be a safe and effective therapy to relieve muscle spasticity after brain injury.

Also, Hui-chan \& Levin [13] mentioned that Low-intensity repetitive electrical stimulation such as dorsal column and Transcutaneous Electrical Nerve Stimulation (TENS) reportedly decreases spasticity and improves voluntary motor control. However, the mechanisms mediating these effects are unclear. Recent findings suggest that spasticity may be characterized more appropriately by a decrease in the stretch reflex threshold than by an increase in gain.

The result of this study supported by Shaygannejad et al., [14] who performed a study on fifty two patients with MS presenting muscle spasm in the leg. They received a four-week treatment course of either baclofen (10mg twice daily, increasing over three weeks to $25 \mathrm{mg}$ ) or self-applied TENS. They observed that spasticity decreased in both groups with the MAS score at the 4 th week followup was significantly lower in the TENS group than the baclofen group.

Moreover, Kwong et al., [15] evaluated the effectiveness of the application of TENS for improving lower extremity motor recovery in stroke survivors. The results showed that TENS improved walking capacity, as measured by either gait speed or the Timed Up and Go Test it also reduced paretic plantar flexor spasticity, as measured using the Modified Ashworth Scale and Composite Spasticity Scale.

However, the results of this study contradicted with Miller et al., [16] who performed a study to evaluate the effectiveness of TENS on spasticity in MS and, furthermore, to compare two different application times [two weeks of 60 minutes and 8 hours daily of TENS applications $(100 \mathrm{~Hz}$ and 0.125 ms pulse width)].

Outcomes were examined using the Global Spasticity Score (GSS), the Penn Spasm Score (PSS), and a Visual Analogue Scale (VAS) for pain. The results of the study demonstrated that there were no statistically significant differences in the GSS following either 60 minutes or 8 hours daily of TENS. However, the 8-hours application time led to a significant reduction in muscle spasm and pain.

Also, some studies indicated that either increased or unchanged spasticity was found after Electrical Stimulation (ES). Robinson et al., [17] investigated the effects of ES on the spastic limbs of spinal cord-injured patients. Thirty-one subjects underwent a surface ES program, stimulating the quadriceps to perform $20 \mathrm{~min}$ of isometric exercise twice daily, 6 days per week for 4-8 weeks. The results demonstrated a tendency toward increasing spasticity in the subjects undergoing ES.

In the present study, there was a statistically significant increase in the post-treatment mean values of Fugel Scale scores in the study group while there was no significant difference in the mean values of Fugel Scale scores pre and posttreatment in control group.

The results of the current study come in agreement with Au-Yeung \& Hui-Chan [18] who performed a double-blind, randomized, placebocontrolled trial on seventy-three patients $<46$ hours post stroke onset with moderate to severe weakness 
in the arm contralateral to the side of stroke. All subjects received conventional rehabilitation. Twenty-nine received additional electrical stimulation, 21 received additional placebo-electrical stimulation and 23 received conventional rehabilitation only, as control. Electrical stimulation or placeboelectrical stimulation was applied to acupuncture points GB20, LI15, LI11, LI10 and LI4, 60 minutes a day, five days a week, for four weeks. The results showed that the electrical stimulation group had greater improvements than the control group in hand grip and pinch strength at $\mathrm{W} 4$, with the gains maintained at W 12 and W24. In contrast, the placebo-electrical stimulation group did not differ from either the control or the electrical stimulation group. Between-group improvements in ARAT scores from baseline to W24 were not significant. They concluded that: Adding four weeks of electrical stimulation during acute stroke appears to produce greater and longer lasting hand grip and pinch strength improvements than administering conventional rehabilitation alone.

Also, the results of the current study were supported by Chen et al., [19] who performed a study on two hundred fifty patients randomized into two groups: Acupuncture (AG) or No Acupuncture (NAG). Eighteen acupuncture treatment sessions were performed over a 3 -week period. The primary outcome was blindly measured with National Institutes of Health Stroke Scale (NIHSS) at week 1 , week 3 , and week 7 . Secondary outcomes included: Fugl-Meyer Assessment (FMA) for motor function, Bedside Swallowing Assessment (BSA) and Video Fluoroscopic Swallowing Study (VFSS) for swallowing function, the Mini-Mental State Examination (MMSE) and Montreal Cognitive Assessment (MoCA) for cognitive function, and the adverse reaction of acupuncture for safety assessment. This trial showed acupuncture is safe and has additional multi-effect in improving neurologic deficits, swallowing disorder, cognitive impairment, and lower extremity function, but has no significant improvement for upper extremity function during this short-term study period.

Moreover, Wayne et al., [20] performed a study on thirty-three subjects with stroke who had moderate to severe UE functional impairment. Active acupuncture tailored to traditional Chinese medicine diagnoses, including electro acupuncture, or sham acupuncture. Up to 20 treatment sessions over a mean of 10.5 weeks. The main outcome measures: UE motor function, spasticity, grip strength, Range of Motion (ROM), activities of daily living, QOL, and mood. All outcomes were measured at baseline and after treatment. Intention-to-Treat (ITT) anal- yses found no statistically significant differences in outcomes between active and sham acupuncture groups. Analyses of protocol-compliant subjects revealed significant improvement in wrist spasticity and both wrist and shoulder ROM in the active acupuncture group, and improvement trends in UE motor function and digit ROM.

\section{Conclusion:}

On the basis of the present data, it is possible to conclude that: The electroacupuncture (TENS) has a significant effect in reduction of spasticity and improvement of upper extremity function following stroke.

\section{References}

1- CHAITALI MADHUSUDAN KULKARNI C.K.: "Comparison between Electrical Stimulation over Motor Point and TENS over Acupuncture Point in Reducing Spasticity and Improving Function after Stroke: Randomized Clinical Trial". International Journal of Physical Medicine \& Rehabilitation, 2-6, 2014.

2- NOMA T., MATSUMOTO S., SHIMODOZONO M., IWASE Y. and KAWAHIRA K.: Novel Neuromuscular Electrical Stimulation System for the Upper Limbs in Chronic Stroke Patients. American Journal of Physical Medicine \& Rehabilitation, 93 (6): 503-10, 2014

3- WANG B.H., LIN C.L., LI T.M., LIN S.D., LIN J.G. and CHOU L.W.: Selection of acupoints for managing upperextremity spasticity in chronic stroke patients. Clinical Interventions in Aging, 9: 147-56, 2014.

4- ALT MURPHY M., PERSSON H.C., DANIELSSON A., BROEREN J., LUNDGREN-NILSSON $\AA$. and SUNNERHAGEN K.S.: SALGOT-Stroke Arm Longitudinal study at the University of Gothenburg, prospective cohort study protocol. BMC Neurology, 11 (1), 56, 2011.

5- SAHIN N., UGURLU H. and ALBAYRAK I.: The efficacy of electrical stimulation in reducing the post-stroke spasticity: A randomized controlled study. Disability and Rehabilitation, 34 (2): 151-6, 2012.

6- CARLSSON C.P. and SJOLUND B.H.: Acupuncture for chronic low back pain: A randomized placebo-controlled study with long-term follow-up. Clin. J. Pain, 17 (4): 296305,2001 .

7- YAN T. and HUI-CHAN C.: Transcutaneous electrical stimulation on acupuncture points improves muscle function in subjects after acute stroke: A randomized controlled trial. Journal of Rehabilitation, (12): 312-6, 2009.

8- HEIDARI M. and HOSSEINI S.A.: Validity of Modified Ashworth Scale as a Measure of Wrist Spasticity in Stroke Patients, Iranian Rehabilitation Journal, 9 (13): 26-30, 2011.

9- SINGER B. and GARCIA-VEGA J.: The Fugl-Meyer Upper Extremity Scale. Journal of Physiotherapy, 63 (1), 53, 2017.

10- LIM S.M., YOO J., LEE E., KIM H.J., SHIN S., HAN G. and AHN H.S.: Acupuncture for Spasticity after Stroke: A Systematic Review and Meta-Analysis of Randomized Controlled Trials, Hindawi Publishing Corporation Evi- 
dence-Based Complementary and Alternative Medicine, Volume 2015, Article ID 870398 1-12, 2015.

11-DESHMUKH M.K., KUMAR C. and GOYAL M.: Application of Transcutaneous Electrical Stimulation on Lower Limb Acupoints as an Important Adjunctive Tool in Stroke Rehabilitation Program \& its Effects on Spasticity and Functional Ability, International Journal of Physiotherapy and Research, Int. J. Physioth. Res., 2013 (3): 63-9, 2013.

12- ZHAO W., WANG C., LI Z., CHEN L., LI J., CUI W. and ZHAO Y.: Efficacy and safety of transcutaneous electrical acupoint stimulation to treat muscle spasticity following brain injury: A double-blinded, multicenter, randomized controlled trial. PLoS ONE, 10 (2): 1-15, 2015.

13- HUI-CHAN C.W.Y. and LEVIN M.F.: Stretch Reflex Latencies in Spastic Hemiparetic Subjects are Prolonged After Transcutaneous Electrical Nerve Stimulation, Canadian Journal of Neurological Sciences, 20: 97-106, 2017.

14- SHAYGANNEJAD V., JANGHORBANI M., VAEZI A., HAGHIGHI S., GOLABCHI K. and HESHMATIPOUR M.: Comparison of the effect of baclofen and transcutaneous electrical nerve stimulation for the treatment of spasticity in multiple sclerosis, A Journal of Progress in Neurosurgery, Neurology and Neurosciences, Volume 35, 2-7, 2013.

15- KWONG P.W.H. and NG G.Y.F.: Transcutaneous electrical nerve stimulation improves walking capacity and reduces spasticity in stroke survivors: A systematic review and meta-analysis, Clinical Rehabilitation, Vol. 32 (9): 1203 19, 2018.

16- MILLER L., MATTISON P., PAUL L. and WOOD L.: The effects of transcutaneous electrical nerve stimulation (TENS) on spasticity in multiple sclerosis, SAGE Journal, Multiple Sclerosis Journal, 13: 527-33, 2007.

17- ROBINSON C.J., KETT N.A. and BOLAM J.M.: Spasticity in spinal cord injured patients: 2 . Initial measures and long-term effects of surface electrical stimulation. Archives of Physical Medicine and Rehabilitation, 69: $862-8,1988$.

18- AU-YEUNG S.S.Y. and HUI-CHAN C.W.Y.: Electrical acupoint stimulation of the affected arm in acute stroke: A placebo-controlled randomized clinical trial. Clinical Rehabilitation, Vol. 28 (2): 149-58, 2014.

19- CHEN L., FANG J., MA R., GU X., CHEN L., LI J. and XU S.: Additional effects of acupuncture on early comprehensive rehabilitation in patients with mild to moderate acute ischemic stroke: A multicenter randomized controlled trial. BMC Complementary and Alternative Medicine, 16 (1): 226, 2016.

20- WAYNE P.M., KREBS D.E., MACKLIN E.A., SCHNYER R., KAPTCHUK T.J., PARKER S.W. and STASON W.B.: Acupuncture for upper-extremity rehabilitation in chronic stroke: A randomized sham-controlled study. Archives of Physical Medicine and Rehabilitation, 86 (12): 2248-55, 2005.

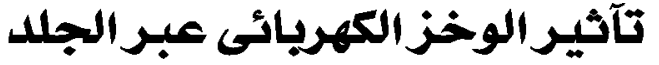 \\ على وظيفة الطرف العلوى لمرضى السكتية الدماغير الجية}

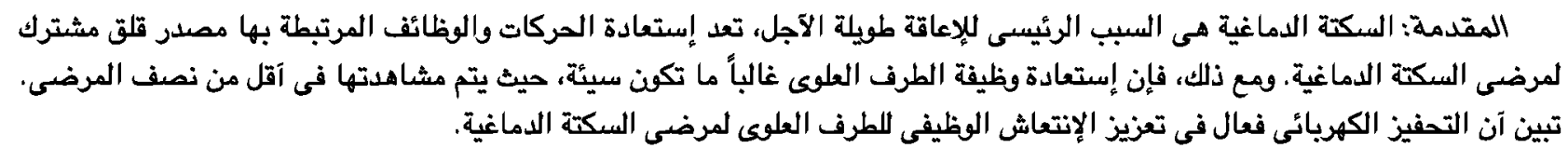

الهدف من الدراسة: لتصديد تآثير التحفيز الكهريائى للعصب عبر الجلد فى نقاط الوخز الإبر على وظيفة الطرف الطلوى فى مرضى السكتة

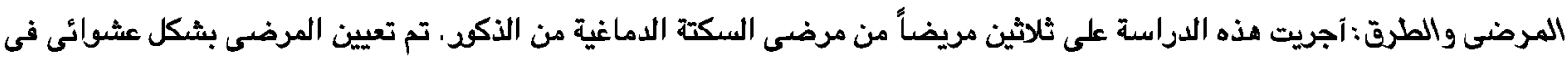

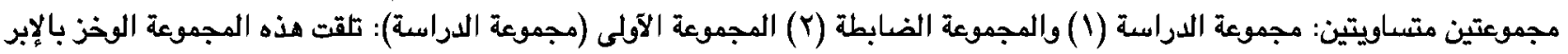

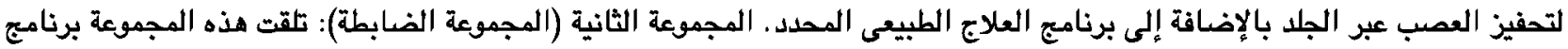

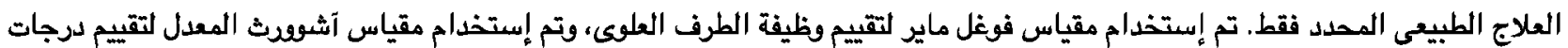
التشنج قبل وبعد آربعة آسابيع من برنامج العبد العلاج.

النتائج: آظهرت النتائج ما يلي: كان هناك إختلاف ذو دلالة إحصائية واضحة فى درجات مقياس فوغل ماير والقيمة المتوسطة لدرجات التشنج فى مجموعة الدراسة بعد برنامج العلاج المان المصمم.

الخلاصة: إن التحفيز الكهربائى للعصب عبر الجلد فت نقاط الوخز الإبر له تآثير كبير فى الحد من التشنج وآيضاً له تآثير كبير فى تصسين وظائف الطرف العلوى بعد السكتة الدماغية. 